\title{
Can placental growth factors explain birthweight variation in offspring of women with type 1 diabetes?
}

\author{
Siobhan Bacon ${ }^{1,2} \cdot$ Dylan Burger $^{3}$ (D) $\cdot$ Mayur Tailor $^{3} \cdot$ J. Johanna Sanchez ${ }^{4}$ (D) George Tomlinson ${ }^{2,5}$. \\ Helen R. Murphy ${ }^{6,7,8}$. Denice S. Feig ${ }^{1,2,9}$ (i) $\cdot$ on behalf of the CONCEPTT Collaborative Group
}

Received: 24 August 2020 / Accepted: 11 December 2020 / Published online: 10 April 2021

(C) The Author(s), under exclusive licence to Springer-Verlag GmbH Germany, part of Springer Nature 2021

\begin{abstract}
Aims/hypothesis Maternal hyperglycaemia alone does not explain the incidence of large offspring amongst women with type 1 diabetes. The objective of the study was to determine if there is an association between placental function, as measured by angiogenic factors, and offspring birthweight $z$ score in women with type 1 diabetes.

Methods This cohort study included samples from 157 Continuous Glucose Monitoring in Pregnant Women with Type 1 Diabetes (CONCEPTT) trial participants. Correlations were estimated between birthweight $z$ score and placental growth factor (PlGF) and soluble fms-like tyrosine kinase (sFlt-1) levels measured at baseline and at 24 and 34 weeks of gestation. Linear regression was used to assess the relationship between birthweight $z$ score and placental health, as measured by PlGF and sFlt-1/P1GF ratio, stratified by glycaemic status (continuous glucose monitoring and $\mathrm{HbA}_{1 \mathrm{c}}$ measures) and adjusted for potential confounders of maternal BMI, smoking and weight gain. Higher PlGF levels and lower sFlt-1/PlGF ratios represent healthy placentas, while lower PlGF levels and higher sFlt-1/PlGF ratios represent unhealthy placentas.

Results Among CONCEPTT participants, the slopes relating PlGF levels to birthweight $z$ scores differed according to maternal glycaemia at 34 weeks of gestation $(p=0.003)$. With optimal maternal glycaemia $\left(\mathrm{HbA}_{1 \mathrm{c}}<48 \mathrm{mmol} / \mathrm{mol}[6.5 \%] / \mathrm{or}\right.$ continuous glucose monitoring time above range $\leq 30 \%$ ), birthweight $z$ scores were reduced towards zero (normal weight) with increasing PlGF values (representing a healthy placenta), and increased with decreasing PlGF values. With suboptimal glycaemic status $\left(\mathrm{HbA}_{1 \mathrm{c}} \geq 48 \mathrm{mmol} / \mathrm{mol}[6.5 \%\right.$ ] or time above range $>30 \%)$, increasing PlGF values were associated with heavier infants. Those with a healthy placenta (PlGF $>100)$ and suboptimal glycaemic control had a higher mean $z$ score $(2.45)$ than those with an unhealthy placenta (mean $z$ score $=1.86$ ). Similar relationships were seen when using sFlt-1/PlGF ratio as a marker for a healthy vs unhealthy placenta.

Conclusions/interpretation In women with type 1 diabetes, infant birthweight is influenced by both glycaemic status and placental function. In women with suboptimal glycaemia, infant birthweight was heavier when placentas were healthy. Suboptimal placental function should be considered in the setting of suboptimal glycaemia and apparently 'normal' birthweight.
\end{abstract}

Keywords Diabetes · Diabetes mellitus · Placenta growth factor · Pregnancy $\cdot$ Pregnancy in diabetics · Pregnancy outcomes $\cdot$ Type 1 diabetes $\cdot$ Vascular endothelial growth factor receptor 1

Denice S. Feig

d.feig@utoronto.ca

1 Mount Sinai Hospital, Sinai Health System, Toronto, ON, Canada

2 Department of Medicine, University of Toronto, Toronto, ON, Canada

3 Department of Cellular and Molecular Medicine, University of Ottawa, Ottawa, ON, Canada

4 Sunnybrook Research Institute, Toronto, ON, Canada
5 University Health Network, Toronto, ON, Canada

6 Cambridge University Hospitals NHS Foundation Trust, Cambridge, UK

7 Norwich Medical School, University of East Anglia, Norwich, UK

8 Women's Health Academic Centre, Division of Women and Children's Health, Kings College London, London, UK

9 Lunenfeld-Tanenbaum Research Institute, Toronto, ON, Canada 


\section{Research in context}

\section{What is already known about this subject?}

- Maternal glycaemia alone does not explain birthweight variation amongst infants of women with type 1 diabetes

- $\quad$ The fetoplacental circulation system impacts on fetal size and is influenced by placental angiogenic factors (PIGF and sFIt-1), with high PIGF or a low sFIt-1/PIGF ratio representing a healthy placenta and low PIGF or a high sFIt$1 /$ PIGF ratio representing an unhealthy placenta

\section{What is the key question?}

- Do alterations in placental angiogenic factors (PIGF and sFlt-1) contribute to variations in infant birthweight amongst women with type 1 diabetes?

What are the new findings?

- Infant birthweight was variably affected by placental angiogenic factors, depending on maternal glycaemia

- Infants of women with suboptimal glycaemia were heavier when placentas were healthy (high PIGF or a low sFlt$1 /$ PIGF ratio), independent of maternal $\mathrm{BMI}$, smoking and weight gain

- Infants of women with suboptimal glycaemia were lighter when placentas were less healthy (low PIGF or a high sFIt-1/PIGF ratio)

\section{How might this impact on clinical practice in the foreseeable future?}

- Additional obstetric surveillance may be applicable in women with $\mathrm{HbA}_{1 c} \geq 48 \mathrm{mmol} / \mathrm{mol}(6.5 \%)$ or $>30 \%$ time above range ( $7.8 \mathrm{mmol} / \mathrm{l}[140 \mathrm{mg} / \mathrm{dl}]$ ) and apparently 'normal' fetal growth, as this may infer suboptimal placental function. More frequent obstetric surveillance (for example, utilising Doppler artery studies) in this group may be appropriate

$\begin{array}{ll}\text { Abbreviations } & \\ \text { CGM } & \text { Continuous glucose monitoring } \\ \text { CONCEPTT } & \text { Continuous Glucose Monitoring in Pregnant } \\ & \text { Women with Type 1 Diabetes trial } \\ \text { GROW } & \text { Gestation-related optimal weight } \\ \text { LGA } & \text { Large for gestational age } \\ \text { NICU } & \text { Neonatal intensive care unit } \\ \text { NPV } & \text { Negative predictive value } \\ \text { PIGF } & \text { Placental growth factor } \\ \text { sFlt-1 } & \text { Soluble fms-like tyrosine kinase } \\ \text { SGA } & \text { Small for gestational age } \\ \text { TAR } & \text { Time above range } \\ \% \text { TAR } & \text { Percentage of time spent above the range } \\ \text { TIR } & \text { Time in range } \\ \text { \%TIR } & \text { Percentage of time spent in range } \\ \text { VEGF } & \text { Vascular endothelial growth factor }\end{array}$

\section{Introduction}

The determinants of fetal growth include genetic make-up and maternal nutrient availability, as well as the capacity of the placenta to adequately transfer both oxygen and nutrients to the fetus and their endocrine modulation [1]. Women with type 1 diabetes have a higher risk of having a baby that is large for gestational age (LGA) or macrosomic [2]. The hypothesis that maternal hyperglycaemia results in fetal hyperinsulinaemia and consequently LGA or macrosomia is undisputed [3]. However, it is long recognised that maternal hyperglycaemia per se is not the only factor contributing to birthweight variation amongst offspring of women with type 1 diabetes. From clinical observation, even women with optimal glycaemic status can have offspring that are classified as LGA or macrosomic [4].

Placental weight correlates with neonatal weight at birth [5-7]. Recently, there has been renewed interest in placental growth factors (PlGFs) and their contribution to the development of preeclampsia and the consequent effect on fetal size. Fetal size is influenced by the fetoplacental circulatory system which begins forming in early gestation and develops throughout pregnancy. PIGFs contribute to the development of the fetoplacental circulatory system. P1GF is a proangiogenic factor secreted by the placenta, which reaches peak concentration at approximately 30 weeks of gestation and then declines [8]. PlGF exerts its pro-angiogenic effects largely through enhancing the effects of vascular endothelial growth factors (VEGFs) [9, 10]. Soluble fms-tyrosine kinase (sFlt-1) is a splice variant of Flt (a VEGF receptor) which acts 
as an antiangiogenic factor by reducing free serum levels of both PlGF and VEGF [11].

Studies of placental factors have largely been in the context of preeclampsia, where low PlGF $(<100 \mathrm{pg} / \mathrm{ml})$ and/or a high sFlt-1/PlGF ratio $(>85)$ are useful as predictors of impending preeclampsia [12]. Women who develop preeclampsia tend to have growth-restricted offspring. Studies in pregnant women without diabetes have also shown an association between low PlGF levels and infants that are small for gestational age (SGA), even in the absence of preeclampsia [13-16]. There are limited data on the association between PlGFs and birthweight $z$ score in women with large babies, and very limited data on women with diabetes. Our aims were: first, to assess the association between PlGFs and birthweight $z$ score in offspring of women with type 1 diabetes; and second, to determine if this association was altered by maternal glycaemic status.

\section{Methods}

\section{Study design and population}

This PlGF study was a secondary analysis of the Continuous Glucose Monitoring in Pregnant Women with Type 1 Diabetes (CONCEPTT) trial. The details of the CONCEPTT trial have been previously published [4]. In brief, CONCEPTT was a multicentre randomised controlled trial of real-time continuous glucose monitoring (CGM) in women with type 1 diabetes who were pregnant or planning pregnancy. Only women who were pregnant were included in this study. Pregnant women with type 1 diabetes aged between 18 and 40 years, with a duration of diabetes $>1$ year and under 14 weeks of gestation, were randomised to capillary glucose monitoring either with or without CGM. The primary outcome was the change in $\mathrm{HbA}_{1 \mathrm{c}}$ from randomisation to 34 weeks of gestation. In addition, other CGM measures of glycaemic control, such as percentage of time in range (\% TIR (3.5-7.8 $\mathrm{mmol} / 1[63-140 \mathrm{mg} / \mathrm{dl}])$ and percentage of time above range (TAR) (\% TAR $>7.8 \mathrm{mmol} / 1$ [140 mg/dl]), were obtained at baseline (approximately 12 weeks) and at 24 and 34 weeks of gestation in both groups. Women were eligible for this study if they had blood samples taken for PlGFs at approximately 12, 24 and 34 weeks of gestation.

\section{Ethics}

The CONCEPTT trial was approved by the Health Research Authority, East of England Research Ethics Committee (12/ $\mathrm{EE} / 0310$ ) for all UK sites and at each individual centre for all other sites. All participants provided written, informed consent. The PlGF study was approved by the Mount Sinai Hospital Research Ethics Board.

\section{Laboratory assays}

For measurement of circulating PlGFs, blood was collected into BD Vacutainer Serum Separator Tubes (Becton Dickinson, USA), allowed to clot for $30 \mathrm{~min}$ at room temperature, centrifuged within $2 \mathrm{~h}$ of collection and transferred to cryovials for storage at $-80^{\circ} \mathrm{C}$ prior to analysis. PlGF and sFlt1 were analysed using commercially available ELISAs (R\&D Systems, Minneapolis, MN, USA) according to the manufacturer's instructions. The interassay coefficients of variation were $7.2 \%$ and $5.6 \%$ for PlGF and sFlt-1, respectively. All $\mathrm{HbA}_{1 \mathrm{c}}$ measurements were performed at a central laboratory (Dynacare, Brampton, ON, Canada) using the turbidimetric inhibition immunoassay for haemolysed whole blood on the Cobas Integra 700 platform (Roche, Basel, Switzerland).

\section{Definitions and outcome measures}

Optimal glucose control was defined as $\mathrm{HbA}_{1 \mathrm{c}}<48 \mathrm{mmol} /$ mol $(6.5 \%)$, and suboptimal glucose control as $\mathrm{HbA}_{1 \mathrm{c}} \geq$ $48 \mathrm{mmol} / \mathrm{mol}$ (6.5\%), CGM percentage time above range (\% TAR $>7.8 \mathrm{mmol} / 1$ [140 mg/dl]) was dichotomised as optimal ( $\leq 30 \%$ TAR) and suboptimal ( $>30 \%$ TAR). Gestational weight gain was calculated from randomisation to 34 weeks and used as a continuous variable. BMI was calculated from enrolment and used as a categorical variable divided into three categories: normal BMI $<25 \mathrm{~kg} / \mathrm{m}^{2}$, overweight 25 to $<30 \mathrm{~kg}$ / $\mathrm{m}^{2}$, obese $\geq 30 \mathrm{~kg} / \mathrm{m}^{2}$.

We utilised high PIGF and a low sFlt-1/P1GF ratio as a surrogate for a 'healthy' placenta. Pathological injury to the placenta resulting in fetal growth restriction occurs via an ischaemia-reperfusion insult to the developing placenta cells. Persistent ischaemia-reperfusion damage to the placenta causes the histological features of maternal vascular malperfusion. This malperfusion ultimately results in an 'unhealthy placenta' and an excessive production and secretion of sFlt-1 with the suppression of secretion of PlGF. As demonstrated in the literature, abnormal circulation of sFlt-1 and PlGF correlates with the extent of placental malperfusion pathology [17, 18]. This was the basis of our rationale for using PlGF and sFlt-1/PlGF ratio to discriminate between healthy and unhealthy placentas. When we defined these angiogenic factors in a dichotomous fashion, a 'healthy' placenta had a PlGF of $>100 \mathrm{pg} / \mathrm{ml}$ or $\mathrm{sFlt}-1 / \mathrm{PlGF}$ ratio of $<85$ and an 'unhealthy' placenta had a PlGF of $<100 \mathrm{pg} / \mathrm{ml}$ or sFlt-1/PlGF ratio of $>85$ [12]. The cut-off for the sFlt-1/ PlGF ratio was based on the study by Verlohren et al [19], whereby, with a cut-off of 85 , the sFlt-1/PlGF ratio yields the highest sensitivity (89\%) and specificity (97\%) for early-onset 
preeclampsia (pre 34 weeks of gestation). Whereas, for the late-onset preeclampsia group ( $>34$ weeks of gestation), a cut-off of 85 results in a sensitivity of $74 \%$ and a specificity of $89 \%$. The cut-off of $100 \mathrm{pg} / \mathrm{ml}$ for PlGF was based on a prospective cohort of 274 women with suspected preeclampsia, whereby a PlGF $<100 \mathrm{pg} / \mathrm{ml}$ had high sensitivity to detect SGA births (sensitivity 93\%, negative predictive value [NPV] 90\%) when compared with fetal weight estimation by ultrasound (sensitivity 71\%, NPV 79\%) [20].

Birthweight $z$ score was used as the primary outcome in this study. SGA was defined as birthweight $<10$ th centile and LGA was defined as birthweight $>90$ th centile using gestation-related optimal weight (GROW)-customised centiles [21].

The neonatal composite outcome was modified from CONCEPTT, and included birth injury, shoulder dystocia, neonatal hypoglycaemia, hyperbilirubinaemia, respiratory distress syndrome or neonatal care admission, but excluded pregnancy loss (miscarriage, stillbirth or neonatal death), as we were interested in the outcome of birthweight.

\section{Statistical analysis}

The main analyses were restricted to those with $\mathrm{HbA}_{1 \mathrm{c}}$, PlGF and sFlt-1/PlGF ratio measured at 34 weeks and a livebirth infant. Scatterplots and Spearman correlations were used to assess univariate relationships of infant birthweight $z$ score with PIGF and the sFIT-1/PIGF ratio after stratifying by maternal glycaemic control. Linear regression models were used to assess whether the relationship between birthweight $z$ score and the growth factors differed according to maternal antenatal glycaemic control. One model was run for PlGF as the measure of placental health and another with the sFIT-1/ PlGF ratio as the measure of placental health. In these models, birthweight $z$ score was the outcome and the predictors were smoking, maternal BMI (modelled with a three-degree of freedom natural spline to account for nonlinearity), maternal weight gain, the logarithm of the growth factor, $\mathrm{HbA}_{1 \mathrm{c}}$ group (optimal $\mathrm{HbA}_{1 \mathrm{c}}<48 \mathrm{mmol} / \mathrm{mol}$ [6.5\%], suboptimal $\mathrm{HbA}_{1 \mathrm{c}} \geq$ $48 \mathrm{mmol} / \mathrm{mol}[6.5 \%]$ and an interaction between these terms. The interaction represents the difference between the optimal and suboptimal $\mathrm{HbA}_{1 \mathrm{c}}$ groups in the slope relating the birthweight $z$ score to the PlGF. This analysis was repeated replacing $\mathrm{HbA}_{1 \mathrm{c}}$ by $\%$ TAR (>7.8 $\left.\mathrm{mmol} / 1[140 \mathrm{mg} / \mathrm{dl}]\right)$ as the measure of maternal antenatal glycaemic control, dichotomised as optimal ( $\leq 30 \%$ TAR) and suboptimal ( $>30 \%$ TAR). In exploratory analyses, the effects of maternal $\%$ TAR, $\mathrm{HbA}_{1 \mathrm{c}}$ and growth factors at baseline (12 weeks) and at 24 weeks were also examined. To quantify the relative importance of the predictors in the explanation of variance $\left(\mathrm{R}^{2}\right)$ in birthweight $z$ scores, we used the Lindemann, Merenda and Gold method to partition the total unadjusted $\mathrm{R}^{2}$ [22]. Interpretation of the PlGF-glycaemic interactions was facilitated by the use of box plots of birthweight $z$ scores in four groups categorised as healthy/unhealthy placental function and optimal/suboptimal glucose control. All analyses used $\mathrm{R}$ version 3.6.1 [23].

\section{Results}

\section{Baseline characteristics}

The analytic cohort included 157 CONCEPTT participants with complete data for PlGF and sFlt-1 variables at 34 weeks of gestation and infant birthweight $z$ score. We excluded 92 participants from the original cohort of 249 pregnant women in CONCEPTT [4]: one withdrew before baseline assessment could be obtained, 89 were missing bloods, and, of those 159 women with bloods, one was missing birthweight due to a withdrawal from the study and one had a stillborn infant, leaving 157 women in this cohort. Women who were included were similar to those excluded (electronic supplementary material [ESM] Table 1). The baseline characteristics of the PIGF study cohort are presented in Table 1. Baseline characteristics according to glycaemic measures can be found in ESM Table 2.

The results are presented in groups categorised as healthy or unhealthy PlGF levels (PIGF $>$ or $\leq 100 \mathrm{pg} / \mathrm{ml}$ and sFlt-1/ PlGF ratio $>$ or $\leq 85$ ). Of note, the duration of diabetes and $\mathrm{HbA}_{1 \mathrm{c}}$ at randomisation and at 34 weeks of gestation were similar across all groups. The percentage of women with microvascular complications also did not differ between the groups. Women with a low PIGF (and high ratio) had more gestational hypertension but less chronic hypertension, and rates of preeclampsia were similar across all groups. The percentage of infants categorised as SGA and LGA was similar across all groups when glycaemic control was not considered.

\section{PIGF and infant birthweight $z$ score by maternal glycaemic status}

Overall, there was a small correlation between PlGF and infant birthweight $z$ score $(\mathrm{r}=-0.08 ; 95 \% \mathrm{CI}-0.08,0.24$, at 34 weeks of gestation). However, the slopes relating PlGF levels to birthweight $z$ scores differed according to maternal glycaemia as assessed by $\mathrm{HbA}_{1 \mathrm{c}}$ at 34 weeks of gestation, after adjusting for maternal BMI, smoking and maternal weight gain $(p=0.003)$ (Fig. 1, ESM Table 3).

In the setting of optimal maternal glycaemia $\left(\mathrm{HbA}_{1 \mathrm{c}}<\right.$ $48 \mathrm{mmol} / \mathrm{mol}[6.5 \%]$ and $\%$ TAR $\leq 30 \%$ ), birthweight $z$ scores were reduced towards zero (normal weight) with increasing PlGF values (representing a healthy placenta). The mean birthweights and $z$ scores were lower (birthweight $=$ $3558 \mathrm{~g} ; z$ score $=1.41$ ) for those with a healthy placenta 
Table 1 Baseline characteristics of the study population, dichotomised into high/low PlGF and sFLT/PlGF at 34 weeks of gestation

\begin{tabular}{|c|c|c|c|c|c|}
\hline Characteristic & All women included & $\mathrm{PlGF}>100 \mathrm{pg} / \mathrm{ml}$ & $\mathrm{PlGF} \leq 100 \mathrm{pg} / \mathrm{ml}$ & sFLT-1/PlGF $\leq 85$ & sFLT-1/PlGF $>85$ \\
\hline$N$ & 157 & 127 & 30 & 128 & 29 \\
\hline Age at entry (years) & $31(4.6)$ & $31.2(4.6)$ & $33.1(4.2)$ & $31(4.6)$ & $33.5(4)$ \\
\hline \multicolumn{6}{|l|}{ BMI category, $n(\%)$} \\
\hline $\operatorname{Normal}\left(<25 \mathrm{~kg} / \mathrm{m}^{2}\right)$ & $82(52.2)$ & $64(50.4)$ & $18(60)$ & $66(51.6)$ & $16(55.2)$ \\
\hline Overweight ( 25 to $<30 \mathrm{~kg} / \mathrm{m}^{2}$ ) & $54(34.4)$ & $44(34.6)$ & $10(33.3)$ & $43(33.6)$ & $11(37.9)$ \\
\hline Obese $\left(\geq 30 \mathrm{~kg} / \mathrm{m}^{2}\right)$ & $21(13.4)$ & $19(15)$ & $2(6.7)$ & $19(14.8)$ & $2(6.9)$ \\
\hline T1D duration (years), median (IQR) & $15(11-22)$ & $15(11-22)$ & $16(12-23)$ & $15(11-22)$ & $16(12-20)$ \\
\hline Gestational age at birth (weeks) & $37.2(1.4)$ & $37.3(1.3)$ & $36.5(1.6)$ & $37.3(1.4)$ & $36.6(1.5)$ \\
\hline \multicolumn{6}{|l|}{$\mathrm{HbA}_{1 \mathrm{c}}$ at randomisation } \\
\hline $\mathrm{mmol} / \mathrm{mol}$ & $51.5(6.2)$ & $51.6(5.7)$ & $51.5(6.3)$ & $51.4(6.3)$ & $51.8(5.7)$ \\
\hline$\%$ & $6.9(0.6)$ & $6.9(0.6)$ & $6.9(0.5)$ & $6.9(0.6)$ & $6.9(0.5)$ \\
\hline \multicolumn{6}{|l|}{$\mathrm{HbA}_{1 \mathrm{c}}$ at 34 weeks } \\
\hline $\mathrm{mmol} / \mathrm{mol}$ & $47.0(7.0)$ & $48.1(5.8)$ & $46.7(7.2)$ & $46.6(7.3)$ & $48.5(5.4)$ \\
\hline$\%$ & $6.5(0.6)$ & $6.4(0.7)$ & $6.6(0.5)$ & $6.4(0.7)$ & $6.6(0.5)$ \\
\hline \multicolumn{6}{|l|}{ Diabetes complications, $n(\%)$} \\
\hline Retinopathy & $30(19.1)$ & $25(19.7)$ & $5(16.7)$ & $25(19.5)$ & $5(17.2)$ \\
\hline Nephropathy & $2(1.3)$ & $2(1.6)$ & $0(0)$ & $2(1.6)$ & $0(0)$ \\
\hline Neuropathy & $4(2.5)$ & $2(1.6)$ & $2(6.7)$ & $2(1.6)$ & $2(6.9)$ \\
\hline Chronic hypertension & $5(3.2)$ & $5(3.9)$ & $0(0)$ & $5(3.9)$ & $0(0)$ \\
\hline Preeclampsia, $n(\%)$ & $17(10.8)$ & $14(11)$ & $3(10)$ & $13(10.2)$ & $4(13.8)$ \\
\hline Gestational hypertension, $n(\%)$ & $17(10.8)$ & $7(5.5)$ & $10(33.3)$ & $6(4.7)$ & $11(37.9)$ \\
\hline Birthweight (g) & $3650(648)$ & $3688(639)$ & $3489(673)$ & $3677(645)$ & $3533(660)$ \\
\hline Birthweight $z$ score & $1.87(1.52)$ & $1.88(1.41)$ & $1.87(1.55)$ & $1.87(1.54)$ & $1.89(1.43)$ \\
\hline $\mathrm{SGA}^{\mathrm{a}}, n(\%)$ & $0(0)$ & $0(0)$ & $0(0)$ & $0(0)$ & $0(0)$ \\
\hline $\mathrm{LGA}^{\mathrm{b}}, n(\%)$ & $102(65.0)$ & $82(64.6)$ & $20(66.7)$ & $83(64.8)$ & $19(65.5)$ \\
\hline
\end{tabular}

Data are mean (SD) unless otherwise stated

${ }^{\text {a }}$ SGA is defined as birthweight $z$ score $<10$ th percentile as calculated using customised birthweight $z$ score percentiles (GROW)

${ }^{\mathrm{b}}$ LGA is defined as birthweight $z$ score $>90$ th percentile as calculated using customised birthweight $z$ score percentiles (GROW)

T1D, type 1 diabetes

compared with those with an unhealthy placenta by PlGF (birthweight $=3619 \mathrm{~g} ; z$ score $=1.93$ ) (Fig. 2).

However, in women with suboptimal glycaemia $\left(\mathrm{HbA}_{1 \mathrm{c}} \geq\right.$ $48 \mathrm{mmol} / \mathrm{mol}$ [6.5\%] and \% TAR $>30 \%$ ), those with healthy placental function (higher PlGF) had heavier offspring (birthweight $z$ scores) than those with lower PlGF (Figs 1, 2 ). In women with suboptimal glycaemia, the mean birthweights and $z$ scores were higher (birthweight $=3853 \mathrm{~g}$; $z$ score $=2.45$ ) for those with a healthy placenta than for those with an unhealthy placenta (birthweight $=3415 \mathrm{~g} ; z$ score $=$ 1.86) by PIGF.

When $\mathrm{HbA}_{1 \mathrm{c}}$ at 34 weeks is divided into three categories reflecting excellent, near-optimal and suboptimal glycaemia, as $\mathrm{HbA}_{1 \mathrm{c}}$ increases, the relationship between PlGF and birthweight $z$ score increases (ESM Fig. 1). This difference in the slopes was not found for $\mathrm{HbA}_{1 \mathrm{c}}$ and PlGF levels at 12 and 24 weeks of gestation (data not shown). The contribution of these covariates to the unadjusted $\mathrm{R}^{2}$ for birthweight $z$ score was $18.7 \%$ overall, with BMI, smoking and gestational weight gain making up $8.9 \%$, and the contribution of $\mathrm{HbA}_{1 \mathrm{c}}, \log$ (PlGF) and the interaction of PlGF and $\mathrm{HbA}_{1 \mathrm{c}}$ group making up 9.8\%. Similar relationships between PlGF levels and birthweight $z$ score when stratified for glycaemic status were seen in preterm $(<37$ weeks) and term $(\geq 37$ weeks) infants at 34 weeks (ESM Fig. 2).

A similar difference was found in the slopes of the relationship between PlGF levels and birthweight $z$ score when stratified by $\%$ TAR $\leq$ or $>30 \% ; p=0.049$ at 34 weeks (Fig. 1, ESM Table 4). This was not seen at 12 or 24 weeks of gestation (data not shown).

\section{sFlt-1/PIGF ratio and infant birthweight $z$ score by maternal glycaemic status}

Similar to PlGF values, there was only a small correlation between sFlt-1/PlGF ratio and infant birthweight $z$ score $(\mathrm{r}=$ 
Fig. 1 Linear model of PlGF at week 34 and offspring birthweight $z$ score by maternal glycaemic status with linear fit adjusted for maternal BMI, smoking and maternal weight gain. Maternal glycaemic status is defined as (a) optimal $\mathrm{HbA}_{1 \mathrm{c}}$ $<48 \mathrm{mmol} / \mathrm{mol}$ or $<6.5 \%$ or (b) suboptimal $\mathrm{HbA}_{1 \mathrm{c}} \geq 48 \mathrm{mmol} / \mathrm{mol}$ or $\geq 6.5 \%$, and using $\%$ TAR $7.8 \mathrm{mmol} / \mathrm{l}(140 \mathrm{mg} / \mathrm{dl})$ as $(\mathbf{c})$ optimal $\leq 30 \%$ or (d) suboptimal $>30 \%$
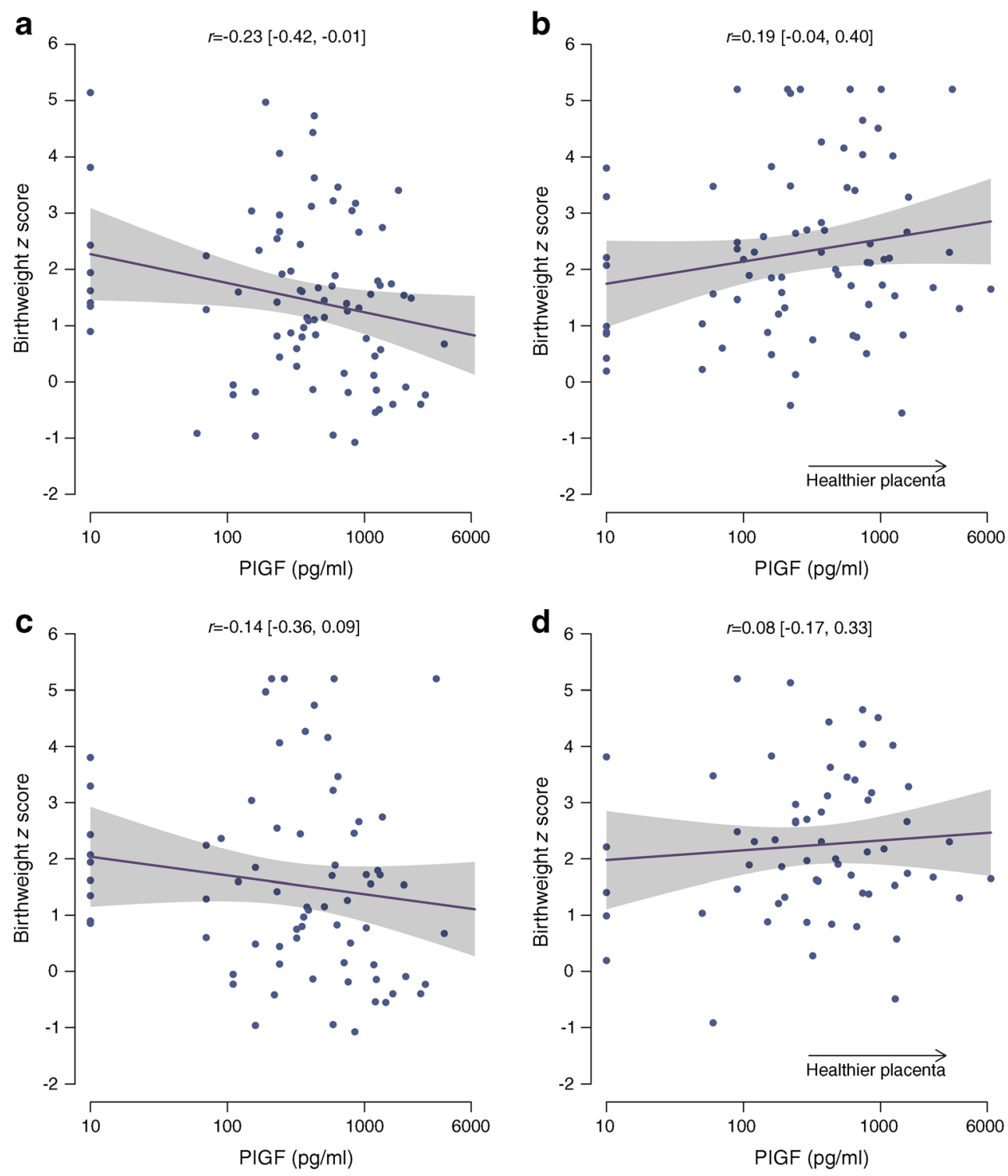

$0.18 ; 95 \%$ CI $0.01,0.32$, at 34 weeks of gestation). However, the slope of the relationship of the sFlt-1/PlGF ratio and mean birthweight $z$ score differed significantly according to maternal glycaemic status by $\mathrm{HbA}_{1 \mathrm{c}}$ after adjusting for maternal BMI, smoking and gestational weight gain $(p=0.0007)$ (Fig. 3, ESM Fig. 3, ESM Table 5).

Similar to PlGF values alone, women with optimal glucose levels $\left(\mathrm{HbA}_{1 \mathrm{c}}<48 \mathrm{mmol} / \mathrm{mol}\right.$ [6.5\%]) and healthy placental function (as measured by sFlt-1/PlGF ratio) had lighter babies (closer to normal weight) compared with those with unhealthy placentas (higher sFlt-1/PlGF ratio). In women with suboptimal glycaemic status $\left(\mathrm{HbA}_{1 \mathrm{c}} \geq 48 \mathrm{mmol} / \mathrm{mol}[6.5 \%]\right)$ those with healthier placentas as measured by a low sFlt-1/PlGF ratio had heavier offspring compared with those with an unhealthy placenta (see Fig. 3, ESM Fig. 3).
The slope of the relationship between the sFlt-1/PlGF ratio and birthweight $z$ score also differed according to glycaemic status as measured by CGM measures (those with $\leq 30 \%$ time spent above the CGM hyperglycaemic target range of $7.8 \mathrm{mmol} / 1$ [140 mg/dl] vs those with $>30 \%$ TAR) (Fig. 3, ESM Table 6) (difference in slopes $p=0.003$ ). For those with near-optimal glycaemic status as evidenced by time above target $<30 \%$, infants were lighter when placentas were healthy. For those with suboptimal glycaemic control with $>30 \%$ of time spent above range, infants were heavier when placentas were healthy (low sFlt-1/PlGF ratio) compared with those with an unhealthy placenta (high sFlt-1/PlGF ratio) (Fig. 3, ESM Fig. 3).

The concordance between the measures of placental function (PlGF and sFlt-1/PlGF ratio) was very good ( $\mathrm{k}$ [kappa statistic $]=0.85 ; 95 \%$ CI $0.75,0.96)$. 
Fig. 2 Box plots depicting birthweight $z$ scores according to low PlGF $(\leq 100 \mathrm{pg} / \mathrm{ml})$ vs high PlGF $(>100 \mathrm{pg} / \mathrm{ml})$ stratified by maternal glycaemic status defined as (a) optimal $\mathrm{HbA}_{1 \mathrm{c}}<48 \mathrm{mmol} /$ mol or $<6.5 \%$ or (b) suboptimal $\mathrm{HbA}_{1 \mathrm{c}} \geq 48 \mathrm{mmol} / \mathrm{mol}$ or $\geq 6.5 \%$ at 34 weeks of gestation, and using \% TAR $7.8 \mathrm{mmol} / 1$ $(140 \mathrm{mg} / \mathrm{dl})$ as $(\mathbf{c})$ optimal $\leq 30 \%$ or (d) suboptimal $>30 \%$
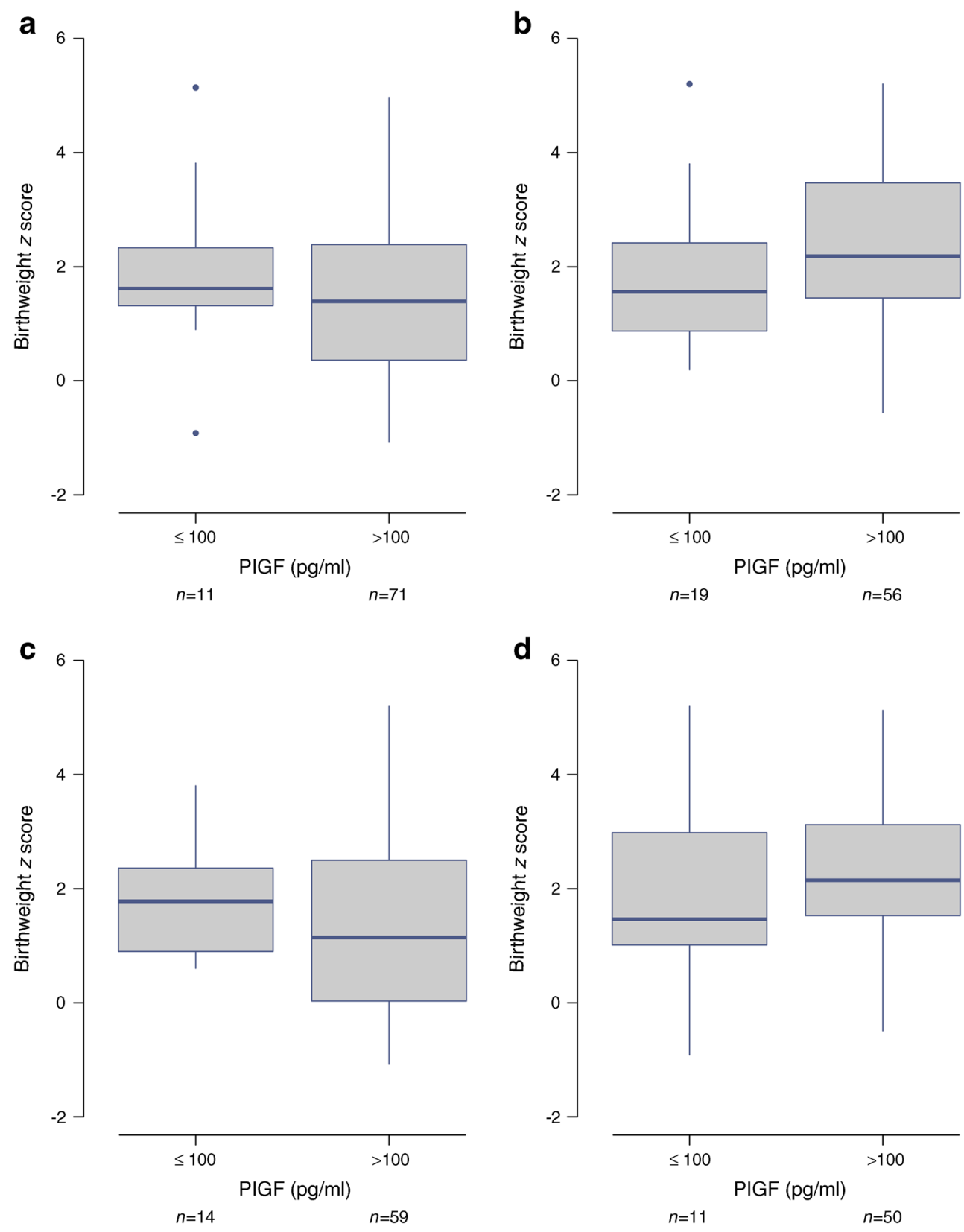

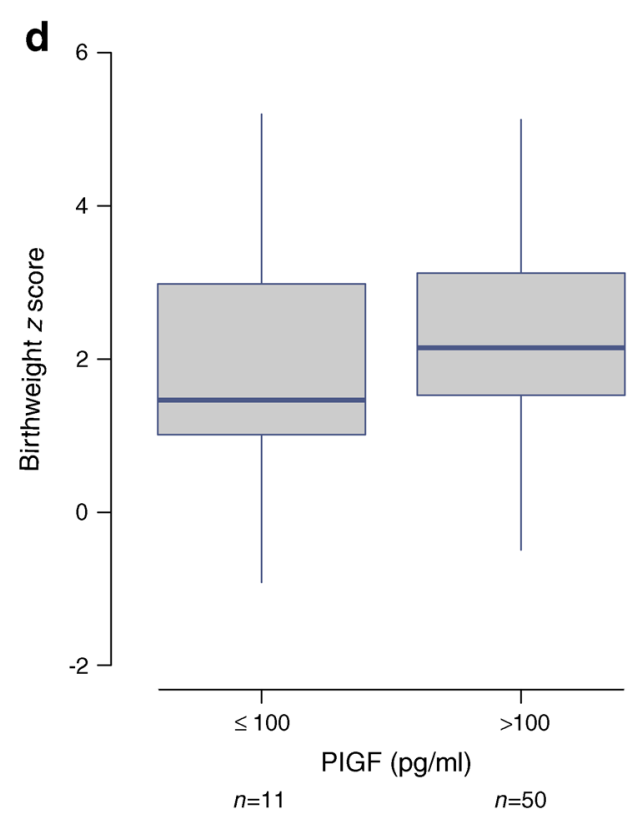

\section{Neonatal outcomes}

The neonatal composite outcome occurred in 7/19 (36.8\%) of the high-HbA $\mathrm{A}_{1 \mathrm{c}}$, low-PlGF group (i.e., with suboptimal glycaemic status and unhealthy placental function) compared with 24/56 (42.9\%) of the high-HbA $\mathrm{H}_{1 \mathrm{c}}$, high-PIGF group (i.e., with suboptimal glycaemic status and healthy placental function at 34 weeks of gestation) $(p=0.85)$.

\section{Discussion}

The offspring of women with type 1 diabetes and suboptimal glycaemic status had heavier birthweight in the setting of placental angiogenic factor levels considered 'healthy' (higher PIGF and lower sFlt-1/PIGF ratio). Conversely, infants of mothers with suboptimal glycaemic status, and placental angiogenic factors considered 'unhealthy' (lower PIGF and high sFlt-1/PIGF ratio), were lighter. The difference was apparent whether glycaemic status was assessed by conventional $\mathrm{HbA}_{1 \mathrm{c}}$ or by $\%$ TAR measures. In women with optimal glucose control the opposite was seen, with healthier placentas associated with lighter infants and unhealthy placental function associated with infants with larger birthweight.

\section{What are the clinical implications of altered PIGFs?}

PIGF as a predictor of macrosomia We found that the heaviest babies were those in mothers with poor glycaemic status and 
Fig. 3 Linear model of sFlt-1/ PlGF ratio at week 34 and offspring birthweight $z$ score by maternal glycaemic status with linear fit adjusted for maternal BMI, smoking and maternal weight gain. Maternal glycaemic status is defined as (a) optimal $\mathrm{HbA}_{1 \mathrm{c}}<48 \mathrm{mmol} / \mathrm{mol}$ or $<6.5 \%$ or (b) suboptimal $\mathrm{HbA}_{1 \mathrm{c}} \geq$ $48 \mathrm{mmol} / \mathrm{mol}$ or $\geq 6.5 \%$, and using \% TAR $7.8 \mathrm{mmol} / \mathrm{l}$ $(140 \mathrm{mg} / \mathrm{dl})$ as (c) optimal $\leq 30 \%$ or (d) suboptimal $>30 \%$
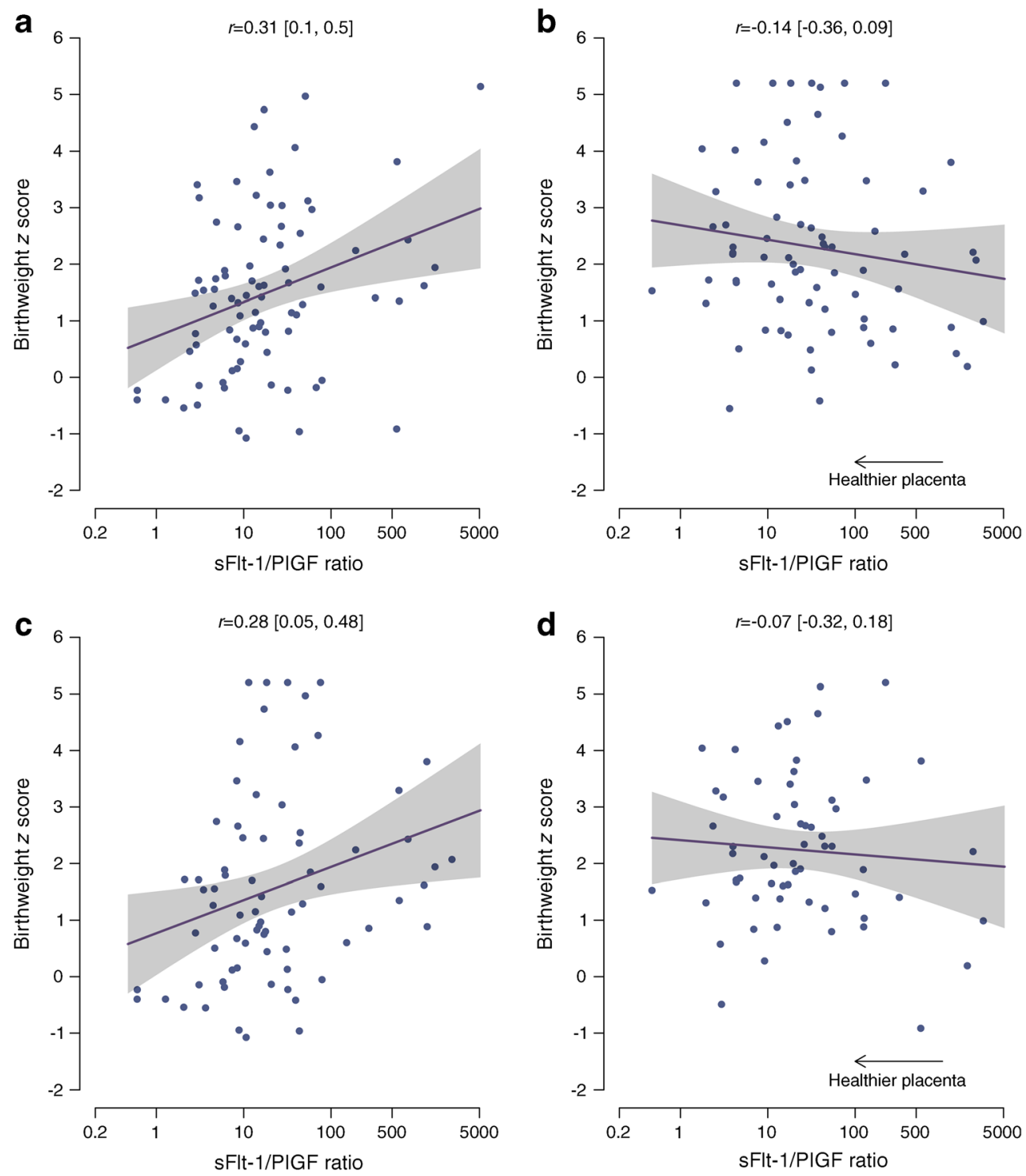

healthy placentas. Transplacental glucose transfer is dependent upon not only the glucose concentration gradient from mother to fetus, but also the uteroplacental blood flow [24, 25]. PlGF, sFlt-1 and VEGF all regulate the development of the placental vascular system. We speculate that an elevation of PIGF (and/or a low sFlt-1/PlGF ratio) may increase placental vascularisation and increase glucose-transporting capacity and the transfer of other fuels, resulting in larger babies. On the other hand, reductions in PlGF (or elevations in sFlt-1/ PIGF ratio) may result in placental dysfunction with reduced transfer of fuels and smaller babies. Several studies have shown a strong association between low PlGF levels and small babies in women without diabetes [14-16]. Few studies, however, have looked at these associations between PlGFs and birthweight in women with diabetes. Kuc et al found that normal levels of PlGF were associated with macrosomia, whereas lower levels were associated with infants of normal size, suggesting a role for PlGF in the size of the baby [26]. James-Todd et al found that third-trimester PIGF levels were significantly associated with birthweight and that high PlGF in the third trimester was associated with an increased risk of macrosomia [27]. They found no association between levels of PlGF and $\mathrm{HbA}_{1 \mathrm{c}}$ in women with type 1 (75\%) and type 2 (25\%) diabetes. It is unfortunate that levels of PlGF and sFit-1/ PIGF ratio stratified by $\mathrm{HbA}_{1 \mathrm{c}}$ were not helpful earlier than 34 weeks. Further work in this area with larger sample sizes may allow better use of these angiogenic markers earlier in pregnancy.

To the best of our knowledge, no previous studies have looked at the association between placental function and infant birthweight stratified for maternal glycaemic status. We found that third-trimester PIGF levels were associated with a bigger baby in the context of suboptimal glucose status and a 'healthy' placenta. In women with suboptimal 
glycaemic status and a healthy placenta (elevated PIGF or low sFlt-1/PlGF ratio), aiming for stricter glucose targets $\left(\mathrm{HbA}_{1 \mathrm{c}}\right.$ $<42 \mathrm{mmol} / \mathrm{mol}[6 \%]$ ) and less than $25 \%$ of time spent above the CGM target range $(7.8 \mathrm{mmol} / 1$ [140 $\mathrm{mg} / \mathrm{dl}])$ may be beneficial in reducing accelerated fetal growth $[28,29]$. In women with optimal glycaemic control, a healthy placenta was associated with a more normal birthweight $z$ score, but an unhealthy placenta was associated with a larger baby. The cause for this is unknown.

PIGF and fetal growth restriction Not all women with suboptimal glycaemic status have a large baby. We have shown that in women with above-target $\mathrm{HbA}_{1 \mathrm{c}}$ level and low PlGF, infants were smaller. These babies, although not meeting clinical definitions of intrauterine growth restriction (IUGR < $2500 \mathrm{~g})$ or SGA $(<10$ th birthweight centile), are still potentially growth restricted in the setting of maternal hyperglycaemia. It has been shown that low third-trimester PIGF can distinguish placenta-mediated fetal growth restriction, detected post hoc by placental lesions indicative of under-perfusion [13]. This may be the case in our 'smaller' infants born to mothers with suboptimal glucose status and 'unhealthy' placentas as measured by angiogenic markers. Fetal growth restriction is associated with multiple neonatal complications including prolonged neonatal intensive care unit (NICU) admissions, hypoglycaemia, infection and respiratory distress [30].

In addition, low PlGF levels are associated with other adverse pregnancy outcomes in women without diabetes. In their meta-analysis, Sherrell et al found an association between low PlGF levels and Caesarean section for fetal compromise, NICU admission and stillbirth [13]. With only 19 infants in the high-HbA 1 , low-PlGF group, we did not have sufficient numbers to evaluate adverse neonatal effects. A larger cohort would be necessary to examine the effect of an 'unhealthy' placenta, particularly in normotensive women with type 1 diabetes. More frequent obstetric surveillance (for example, utilising Doppler artery studies) may be appropriate. More women in the 'unhealthy' placenta group had gestational hypertension, which may have been related to placental insufficiency and have played a role in the smaller babies. However, this effect was more prominent in those with suboptimal glucose status, suggesting that gestational hypertension may not be as detrimental on birthweight $z$ score in women with optimal glycaemia.

Our study has several strengths. One is the wellcharacterised cohort of pregnant women with type 1 diabetes who were followed prospectively with detailed glycaemic measures using both $\mathrm{HbA}_{1 \mathrm{c}}$ and CGM. It was performed across 31 centres in six countries and data are therefore generalisable to many healthcare settings. We were able to evaluate PlGF and sFlt-1 in each trimester and to correlate with infant birthweight $z$ score, stratified for maternal glycaemic status. There are also some limitations. We were unable to obtain blood samples from all CONCEPTT participants. With a larger sample size, we may have been able to explore whether the potential 'growth-restricted' infants had other neonatal complications and to assess the influence of different PlGF levels and sFlt-1/PlGF ratios on neonatal outcomes. More studies are needed to understand why women with optimal glycaemic control had poor placental health and bigger babies.

Conclusions In women with type 1 diabetes, infant birthweight is influenced both by maternal glycaemic status and by placental function, as measured by PlGF levels and sFlt-1/P1GF ratio. In the future, placental function assessment and additional obstetric surveillance may be appropriate for women with suboptimal glycaemic status and apparently normal fetal growth. Likewise, assessing longitudinal placental function measurements may also be applicable in women with optimal glycaemic status who are predicted to have babies with large birthweight. Measuring PlGF and/or sFlt-1/PlGF ratios in pregnant women with type 1 diabetes may help to predict compromised placental function and reduce perinatal complications. Although these results are intriguing, they are preliminary and future research will help to determine the usefulness of incorporating these measures of placental function into clinical practice outside of their use in predicting preeclampsia.

Supplementary Information The online version contains peer-reviewed but unedited supplementary material available at https://doi.org/10.1007/ s00125-021-05438-y.

Acknowledgements The authors would like to thank all the women with type 1 diabetes who participated in this study. We also acknowledge the invaluable support from the 31 clinical care teams and the CONCEPTT Steering Committee: D. S. Feig (Mount Sinai Hospital, Toronto, Canada), H. R. Murphy (University of East Anglia, Norwich, UK), E. Asztalos (Sunnybrook Research Institute, Toronto, Canada), J. F. R. Barrett (Sunnybrook Research Institute, Toronto, Canada), J. J. Sanchez (Sunnybrook Research Institute, Toronto, Canada), R. Corcoy (Hospital de la Santa Creu i Sant Pau CIBER-BBN, Barcelona, Spain), A. de Leiva (Hospital de la Santa Creu i Sant Pau CIBER-BBN, Barcelona, Spain), L. E. Donovan (University of Calgary, Calgary, Canada), M. Hod (Rabin Medical Center, Petah, Tikvah, Israel), L. Jovanovic (University of Southern California, Los Angeles, USA), E. Keely (University of Ottawa, Ottawa, Canada), C. Kollman (Jaeb Center For Health Research, Tampa, FL, USA), R. McManus (St Joseph Health Care London, London, Canada), K. E. Murphy (Mount Sinai Hospital, Toronto, Canada), K. Ruedy (Jaeb Center For Health Research, Tampa, FL, USA) and G. Tomlinson (University Health Network, Toronto, Canada). Parts of this study were presented in abstract form at the 78th Scientific Sessions of the American Diabetes Association, San Francisco, CA, June 2019. 
Data availability All data generated or analysed during this study are included in this published article (and its supplementary information files)

Funding CONCEPTT was funded by JDRF grant 17-2011-533 and grants under the JDRF Canadian Clinical Trial Network, a publicprivate partnership including JDRF and FedDev Ontario and supported by JDRF no. 80-2010-585. Medtronic supplied the CGM sensors and CGM systems at a reduced cost. HRM conducts independent research supported by the National Institute for Health Research (Career Development Fellowship CDF-2013-06-035). JJS, GT, HRM and DSF report grants from JDRF. HRM is supported by Tommy's charity. DSF reports grants from the Canadian Institutes of Health Research. DB is supported by funding from the Canadian Institutes of Health Research and an Ontario Early Researcher Award. The views expressed in this publication are those of the authors and not necessarily those of the National Institute for Health Research or the UK Department of Health. The study sponsor/funder was not involved in the design of the study; the collection, analysis, and interpretation of data; or the writing of the report; and did not impose any restrictions regarding the publication of the report.

Authors' relationships and activities The authors declare that there are no relationships or activities that might bias, or be perceived to bias, their work.

Contribution statement All authors made substantial contributions to conception and design, acquisition of data, or analysis and interpretation of data; to drafting the article or revising it critically for important intellectual content; and gave final approval of the version to be published. $\mathrm{SB}$, DSF and GT are the guarantors of this work and, as such, had full access to all the data in the study and take responsibility for the integrity of the data and the accuracy of the data analysis.

\section{References}

1. Owens JA (1991) Endocrine and substrate control of fetal growth: placental and maternal influences and insulin-like growth factors. Reprod Fertil Dev 3(5):501-517. https://doi.org/10.1071/ RD9910501

2. Mackin ST, Nelson SM, Kerssens JJ et al (2018) Diabetes and pregnancy: national trends over a 15 year period. Diabetologia 61(5):1081-1088. https://doi.org/10.1007/s00125-017-4529-3

3. Pedersen J (1954) Weight and length at birth of infants of diabetic mothers. Acta Endocrinol 16(4):330-342. https://doi.org/10.1530/ acta. 0.0160330

4. Feig DS, Donovan LE, Corcoy R et al (2017) Continuous glucose monitoring in pregnant women with type 1 diabetes (CONCEPTT): a multicentre international randomised controlled trial. Lancet 390(10110):2347-2359. https://doi.org/10.1016/S0140-6736(17) 32400-5

5. Mayhew TM (2002) Enhanced fetoplacental angiogenesis in pregestational diabetes mellitus: the extra growth is exclusively longitudinal and not accompanied by microvascular remodelling. Diabetologia 45(10):1434-1439. https://doi.org/10.1007/s00125002-0927-1

6. Cvitic S, Desoye G, Hiden U (2014) Glucose, insulin, and oxygen interplay in placental hypervascularisation in diabetes mellitus. Biomed Res Int 2014:145846. https://doi.org/10.1155/2014/ 145846

7. Starikov R, Inman K, Chen K et al (2014) Comparison of placental findings in type 1 and type 2 diabetic pregnancies. Placenta 35(12): 1001-1006. https://doi.org/10.1016/j.placenta.2014.10.008
8. Chau K, Hennessy A, Makris A (2017) Placental growth factor and pre-eclampsia. J Hum Hypertens 31(12):782-786. https://doi.org/ 10.1038/jhh.2017.61

9. Autiero M, Luttun A, Tjwa M, Carmeliet P (2003) Placental growth factor and its receptor, vascular endothelial growth factor receptor1 : novel targets for stimulation of ischemic tissue revascularization and inhibition of angiogenic and inflammatory disorders. J Thromb Haemost 1(7):1356-1370

10. Autiero M, Waltenberger J, Communi D et al (2003) Role of PlGF in the intra- and intermolecular cross talk between the VEGF receptors Flt1 and Flk1. Nat Med 9(7):936-943. https://doi.org/10.1038/ nm884

11. Lim JH, Kim SY, Park SY, Yang JH, Kim MY, Ryu HM (2008) Effective prediction of preeclampsia by a combined ratio of angiogenesis-related factors. Obstet Gynecol 111(6):1403-1409. https://doi.org/10.1097/AOG.0b013e3181719b7a

12. Duhig KE, Myers J, Seed PT et al (2019) Placental growth factor testing to assess women with suspected pre-eclampsia: a multicentre, pragmatic, stepped-wedge cluster-randomised controlled trial. Lancet 393(10183):1807-1818. https://doi.org/10. 1016/S0140-6736(18)33212-4

13. Sherrell H, Dunn L, Clifton V, Kumar S (2018) Systematic review of maternal placental growth factor levels in late pregnancy as a predictor of adverse intrapartum and perinatal outcomes. Eur $\mathrm{J}$ Obstet Gynecol Reprod Biol 225:26-34. https://doi.org/10.1016/j. ejogrb.2018.03.059

14. Wallner W, Sengenberger R, Strick R et al (2007) Angiogenic growth factors in maternal and fetal serum in pregnancies complicated by intrauterine growth restriction. Clin Sci (Lond) 112(1):5157. https://doi.org/10.1042/CS20060161

15. Poon LC, Zaragoza E, Akolekar R, Anagnostopoulos E, Nicolaides $\mathrm{KH}$ (2008) Maternal serum placental growth factor (PlGF) in small for gestational age pregnancy at $11(+0)$ to $13(+6)$ weeks of gestation. Prenat Diagn 28(12):1110-1115. https://doi.org/10.1002/pd. 2143

16. Sundrani D, Khot V, Pisal H et al (2013) Gestation dependant changes in angiogenic factors and their associations with fetal growth measures in normotensive pregnancy. PLoS One 8(1): e54153. https://doi.org/10.1371/journal.pone.0054153

17. Rajakumar A, Cerdeira AS, Rana S et al (2012) Transcriptionally active syncytial aggregates in the maternal circulation may contribute to circulating soluble fms-like tyrosine kinase 1 in preeclampsia. Hypertension 59(2):256-264. https://doi.org/10.1161/ HYPERTENSIONAHA.111.182170

18. Tache V, LaCoursiere DY, Saleemuddin A, Parast MM (2011) Placental expression of vascular endothelial growth factor receptor-1/soluble vascular endothelial growth factor receptor-1 correlates with severity of clinical preeclampsia and villous hypermaturity. Hum Pathol 42(9):1283-1288. https://doi.org/10. 1016/j.humpath.2010.11.018

19. Verlohren S, Galindo A, Schlembach D et al (2010) An automated method for the determination of the sFlt-1/PIGF ratio in the assessment of preeclampsia. Am J Obstet Gynecol 202(2):161 e1- e11

20. Griffin M, Seed PT, Duckworth S et al (2018) Predicting delivery of a small-for-gestational-age infant and adverse perinatal outcome in women with suspected pre-eclampsia. Ultrasound Obstet Gynecol 51(3):387-395. https://doi.org/10.1002/uog. 17490

21. Gardosi J, Clausson B, Francis A (2009) The value of customised centiles in assessing perinatal mortality risk associated with parity and maternal size. BJOG 116(10):1356-1363. https://doi.org/10. $1111 / \mathrm{j} .1471-0528.2009 .02245 . x$

22. Groemping $U$ (2006) Relative importance for linear regression in $R$ : The Package relaimpo. J Stat Softw 17(1). https://www.jstatsoft. org/article/view/v017i01 
23. R Core Team (2019) R: a language and environment for statistical computing. R Foundation for Statistical Computing, Vienna, Austria

24. Hay WW Jr (1991) The placenta. Not just a conduit for maternal fuels. Diabetes 40(Suppl 2):44-50

25. Castillo-Castrejon M, Powell TL (2017) Placental nutrient transport in gestational diabetic pregnancies. Front Endocrinol (Lausanne) 8: 306

26. Kuc S, Wortelboer EJ, Koster MP, de Valk HW, Schielen PC, Visser GH (2011) Prediction of macrosomia at birth in type-1 and 2 diabetic pregnancies with biomarkers of early placentation. BJOG 118(6):748-754. https://doi.org/10.1111/j.1471-0528.2011.02904.

27. James-Todd T, Cohen A, Wenger J, Brown F (2016) Time-specific placental growth factor (PlGF) across pregnancy and infant birth weight in women with preexisting diabetes. Hypertens Pregnancy 35(3):436-446. https://doi.org/10.3109/10641955.2016.1172085
28. Murphy HR (2019) Continuous glucose monitoring targets in type 1 diabetes pregnancy: every $5 \%$ time in range matters. Diabetologia 62(7):1123-1128. https://doi.org/10.1007/s00125-019-4904-3

29. Battelino T, Danne T, Bergenstal RM et al (2019) Clinical targets for continuous glucose monitoring data interpretation: recommendations from the international consensus on time in range. Diabetes Care 42(8):1593-1603. https://doi.org/10.2337/dci19-0028

30. Malhotra A, Allison BJ, Castillo-Melendez M, Jenkin G, Polglase GR, Miller SL (2019) Neonatal morbidities of fetal growth restriction: pathophysiology and impact. Front Endocrinol (Lausanne) 10: 55

Publisher's note Springer Nature remains neutral with regard to jurisdictional claims in published maps and institutional affiliations. 